\title{
ARTICLE
}

\section{Shielding Experiments at High Energy Accelerators of Fermilab(II) - Spatial distribution measurement of reaction rate behind the shield and its application for Moyer model -}

\author{
Hiroshi YASHIMA ${ }^{1 *}$, Yoshimi KASUGAI ${ }^{2}$, Norihiro MATSUDA ${ }^{2}$, Hiroshi MATSUMURA ${ }^{3}$, Hiroshi IWASE ${ }^{3}$, \\ Norikazu KINOSHITA ${ }^{3}$, Nikolai MOKHOV ${ }^{4}$, Anthony LEVELING ${ }^{4}$, David BOEHNLEIN ${ }^{4}$, Kamran VAZILI $^{4}$, \\ Lautenschlager GARY ${ }^{4}$, Schmitt WAYNE ${ }^{4}$, Takashi NAKAMURA ${ }^{5,6}$, Koji OISHI $^{6}$, Hideo HIRAYAMA ${ }^{3}$, \\ Kenji ISHIBASHI $^{7}$, Hiroshi NAKASHIMA ${ }^{2}$, Yukio SAKAMOTO ${ }^{2}$, and members of JASMIN collaboration \\ ${ }^{1}$ Kyoto University Research Reactor Institute, Kumatori-cho, Sennan-gun, Osaka 590-0494, Japan \\ ${ }^{2} J a p a n$ Atomic Energy Agency, 2-4, Shirakata Shirane, Tokai-mura, Ibaraki 319-1195, Japan \\ ${ }^{3}$ High Energy Accelerator Research Organization, 1-1, Oho, Tsukuba, Ibaraki 305-0801, Japan \\ ${ }^{4}$ Fermi National Accelerator Laboratory, Batavia, IL 87545, USA \\ ${ }^{5}$ Cyclotron and Radioisotope Center, Tohoku University, Aoba, Aramaki, Aoba-ku, Sendai, Miyagi 980-8578 Japan \\ ${ }^{6}$ Shimizu Corporation, 4-17, Etchujima 3-chome, Koto-ku, Tokyo 135-8530, Japan \\ ${ }^{7}$ Kyushu University, 744, Motooka, Nishi-ku, Fukuoka 819-0395, Japan
}

JASMIN - Japanese and American Study of Muon Interaction and Neutron detection - a program for studies of shielding and irradiation effect around high energy accelerators has been started until 2007 using high energy proton accelerators located in Fermi National Accelerator Laboratory (FNAL) as a collaboration of JAPAN and FNAL. The series of the presentations entitled "Shielding experiments at high energy accelerators of Fermilab" describes the part of the results of this collaboration regarding transport of secondary particles, neutron and muon, from $120 \mathrm{GeV}$ proton induced reactions through experimental data and simulation. In this study, the spatial distribution of reaction rate of activation samples which were placed on pbar anti proton target station were obtained. The measured data shows that the reaction rates on the outer surfaces of the iron and concrete shields increases toward the downstream of the target. The attenuation length for iron were obtained from depth distribution of reaction rates in iron shield. The obtained reaction rates were also fitted to Moyer's formula. The shape of angular distribution of fitted reaction rates agree with that of measured reaction rates for $\theta=60-120$ degrees.

\section{KEYWORDS: Moyer's formula, Activation Detector, Fermi National Accelerator Laboratory, attenuation length, $120 \mathrm{GeV}$}

\section{Introduction}

The Japanese and American Study of Muon Interaction and Neutron detection (JASMIN) collaboration has been organized to study radiation safety issues around high energy accelerators in $\mathrm{FNAL}^{1)}$. The collaboration aims to study behaviors of secondary particles generated from beam losses in high energy accelerators. The physical quantities which should be measured for this purpose are particle flux, activities in air, water and materials, damage of instruments and so on. The remarkable efforts for similar experiments have been performed until now ${ }^{2,3,4,5}$, however only a few attempt exist more than $1 \mathrm{GeV}$ energy region ${ }^{6,7,8)}$. At around this energy, most simulation codes switch implemented reaction models from one for intermediate to high energy ${ }^{9}$. The methodology of the collaboration includes not only taking experimental data but also benchmark and improvement of the reaction models.

The data takings have been performed using two facilities

*Corresponding Author, E-mail:yashima@rri.kyoto-u.ac.jp

(C) Atomic Energy Society of Japan in FANL, anti proton production (pbar) and neutrino from main injector (NuMI). The pbar is prepared for anti-proton production using $120 \mathrm{GeV}, 65 \mathrm{~kW}$ proton beam, which is required for FNAL collider experiment, Tevatron. The shielding structure of pbar is suitable for this type experiment. The induced activities and neutron flux measured at this facility, and it's simulation will be reported in the successive papers.

In this study, the spatial distribution of reaction rate of samples which were placed in pbar target station were measured to obtain the experimental data for high energy secondary particle transport from target bombarded by high energy proton. The attenuation length for iron were obtained from depth distribution of reaction rates in iron shield. The obtained reaction rates were also fitted to Moyer's formula $^{10,11)}$, which is used for shielding design of high energy accelerator facilities above $1 \mathrm{GeV}$.

\section{Experiment}

Fig. 1 shows the cross sectional view of the Pbar target station. At this station, an antiproton production made of 
inconel and copper disks irradiated by $120 \mathrm{GeV}$ protons. Average beam intensity is $2.2 \times 10^{12}$ protons/sec. In downstream of the target, a correction lens, collimator and a pulsed magnet are placed to focus, collimate, and extract the produced antiprotons. The remaining protons and secondary particles are absorbed by the dump placed in downstream of the pulsed magnet. Iron and concrete shields are placed upper side of the target and magnets. The thickness of iron and concrete above the target are 6 and 4 feet, respectively. Air gap of 6 feet height is between the iron and concrete shields. The distance between target and iron shields is 46 $\mathrm{cm}$. The activation samples were placed behind iron and concrete shields, which were referred to as air gap (AG) samples and concrete cap (CC) samples to measure spatial distribution of secondary neutrons, respectively. The Iron shield were composed of iron plate, and the activation samples were installed in iron plate, which were placed in $11-\mathrm{cm}$ downstream from the target for beam axis, to measure attenuation of secondary neutrons in iron shields. These samples were referred to as filler plate (FP) samples. The physical properties of radioactive nuclides used in this study are listed in Table $\mathbf{1}^{12)}$.

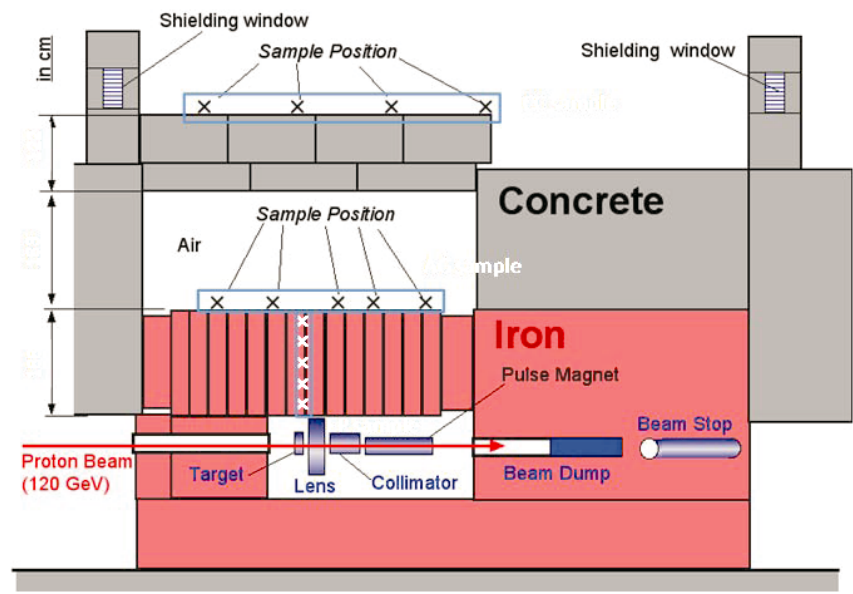

Fig. 1 The cross sectional view of the Pbar target station.

After irradiation, the gamma-rays emitted from the irradiated samples were measured with a high-purity germanium (HPGe) detectors. Reaction rates of radioactive nuclides produced in samples, which were identified in the gamma-ray spectra and the decay curves, were determined after being corrected for the peak efficiency of the HPGe detectors and the beam current fluctuation during the irradiation. One of the HPGe detectors was calibrated by Canberra. The peak efficiency was determined by using the LabSOCS software of Canberra ${ }^{13)}$ for the calibrated HPGe detector, and from the peak counting rate ratios of non-calibrated HPGe detector to the calibrated HPGe detector for non-calibrated HPGe detector. The components of estimated errors in reaction rates were the counting statistics and detector efficiency.
Table 1 The physical properties of radioactive nuclides.

\begin{tabular}{|c|c|c|c|c|c|}
\hline Reaction & $\begin{array}{l}\text { Threshold } \\
{[\mathrm{MeV}]}\end{array}$ & Half & $\begin{array}{c}\text { Gamma-ray } \\
\text { Energy }[\mathrm{keV}]\end{array}$ & $\begin{array}{r}\text { Branching } \\
\text { Ratio[\%] }\end{array}$ & Location \\
\hline${ }^{27} \mathrm{Al}(\mathrm{n}, \alpha)^{24} \mathrm{Na}$ & 3.3 & $14.959 \mathrm{~h}$ & 1368.63 & 100 & CC AG FP \\
\hline${ }^{209} \mathrm{Bi}(\mathrm{n}, 4 \mathrm{n}){ }^{206} \mathrm{Bi}$ & 22.6 & $6.243 \mathrm{~d}$ & 803.1 & 98.9 & CC AG FP \\
\hline${ }^{209} \mathrm{Bi}(\mathrm{n}, 5 \mathrm{n}){ }^{205} \mathrm{Bi}$ & 29.6 & $15.31 \mathrm{~d}$ & 703.45 & 31.1 & CC AG FP \\
\hline${ }^{209} \mathrm{Bi}(\mathrm{n}, 6 \mathrm{n}){ }^{204} \mathrm{Bi}$ & 38 & $11.22 \mathrm{~h}$ & 374.76 & 81.8 & CC AG FP \\
\hline${ }^{209} \mathrm{Bi}(\mathrm{n}, 7 \mathrm{n})^{203} \mathrm{Bi}$ & 45.3 & $11.76 \mathrm{~h}$ & 820.23 & 29.6 & CC AG FP \\
\hline${ }^{115} \operatorname{In}\left(\mathrm{n}, \mathrm{n}^{\prime}\right){ }^{115 \mathrm{~m}} \mathrm{In}$ & 0.6 & $4.486 \mathrm{~h}$ & 336.241 & 45.9 & $\mathrm{CC}$ \\
\hline$\left.{ }^{93} \mathrm{Nb}(\mathrm{n}, 2 \mathrm{n})\right)^{92 \mathrm{~m}} \mathrm{Nb}$ & 9.1 & $10.15 \mathrm{~d}$ & 934.44 & 99.1 & AG FP \\
\hline${ }^{93} \mathrm{Nb}(\mathrm{n}, 4 \mathrm{n}){ }^{90} \mathrm{Nb}$ & 29.1 & $14.6 \mathrm{~h}$ & 1129.224 & 92.7 & AG \\
\hline${ }^{27} \mathrm{Al}(\mathrm{n}, \alpha 2 \mathrm{n}){ }^{22} \mathrm{Na}$ & 23.3 & $2.6019 \mathrm{y}$ & 1274.53 & 99.94 & AG \\
\hline${ }^{\text {nat }} \mathrm{Cu}(\mathrm{n}, \mathrm{x}){ }^{58} \mathrm{Co}$ & & $70.82 \mathrm{~d}$ & 810.78 & 99.448 & $\mathrm{CC}$ AG \\
\hline${ }^{n a t} \mathrm{Cu}(\mathrm{n}, \mathrm{x}){ }^{57} \mathrm{Co}$ & & $271.79 d$ & 122.06 & 85.6 & $\mathrm{CC}$ \\
\hline${ }^{n a t} \mathrm{Cu}(\mathrm{n}, \mathrm{x}){ }^{56} \mathrm{Co}$ & & $77.27 \mathrm{~d}$ & 846.77 & 99.935 & $\mathrm{CC}$ \\
\hline${ }^{\text {nat }} \mathrm{Cu}(\mathrm{n}, \mathrm{x})^{59} \mathrm{Fe}$ & & $44.503 d$ & 1099.25 & 56.5 & $\mathrm{CC}$ \\
\hline${ }^{\text {nat }} \mathrm{Cu}(\mathrm{n}, \mathrm{x}){ }^{54} \mathrm{Mn}$ & & $312.12 \mathrm{~d}$ & 834.85 & 99.976 & $\mathrm{CC}$ \\
\hline${ }^{\text {nat }} \mathrm{Cu}(\mathrm{n}, \mathrm{x})^{52} \mathrm{Mn}$ & & $5.591 \mathrm{~d}$ & 744.2 & 90.6 & $\mathrm{CC}$ \\
\hline
\end{tabular}

\section{Results and discussion}

\section{Spatial distribution of reaction rates of nuclides induced in CC, AG and FP samples}

The reaction rates of nuclides induced in $\mathrm{CC}$ and $\mathrm{AG}$ samples and depth distribution of reaction rate of nuclides induced in FP samples were obtained. The unit of the reaction rate is reaction per nuclide per proton. The measured reactions were listed in Table 1. The spatial distribution of reaction rates of nuclides induced in CC, AG, and FP samples are shown in Figs.3, 4 and5, respectively. Horizontal axis is sample position from target for beam direction in Figs.3 and $\mathbf{4}$ and depth of iron shield in Fig.5. In Figs.3 and 4, the measured data shows that the reaction rates on the outer surfaces of the iron and concrete shields increase toward the downstream of the target for $\mathrm{CC}$ and $\mathrm{AG}$ samples. It means that neutron flux from the target is larger for forward direction from beam axis. In Fig.5, the reaction rate of nuclide induced in FP samples decreases exponentially with depth of iron shield.

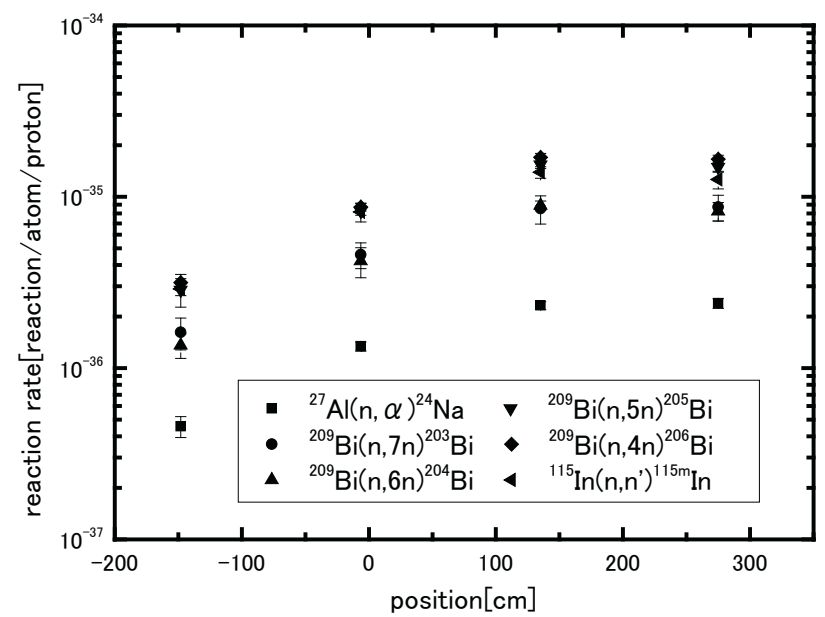

Fig. 3 The spatial distribution of reaction rates of nuclides induced in CC samples 


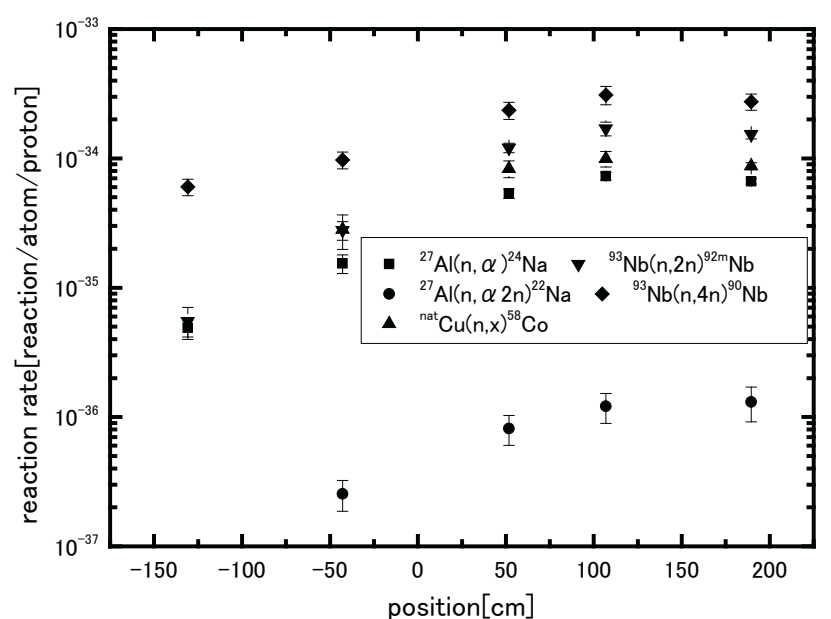

Fig. 4 The spatial distribution of reaction rates of nuclides induced in AG samples

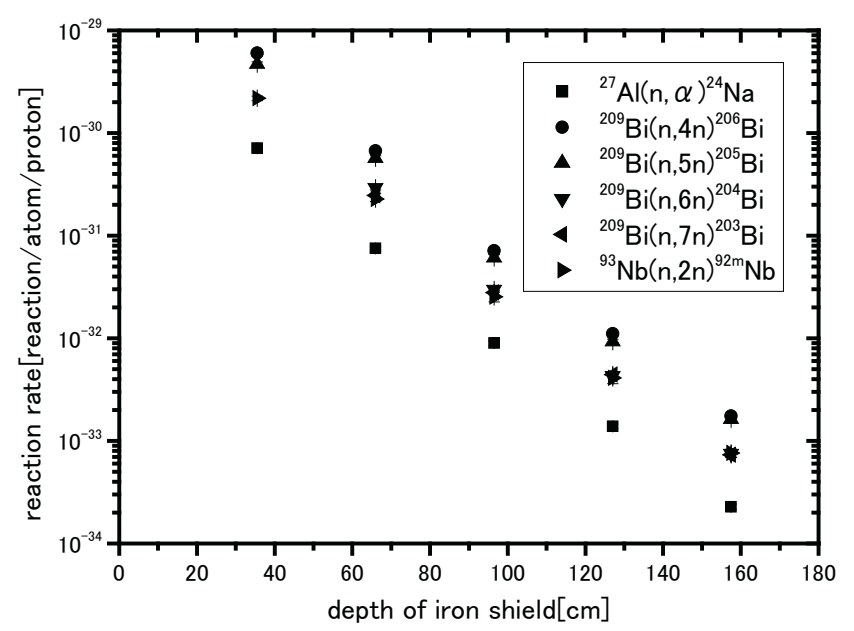

Fig. 5 The spatial distribution of reaction rates of nuclides induced in samples

\section{Determination of attenuation length in iron shield}

The depth distribution of reaction rate of nuclides induced in FP samples indicates neutron attenuation for iron shields. . The attenuation length for iron, $\lambda_{\text {iron}}$, was obtained from the depth distribution of multiplying reaction rate by the square distance between target and samples as shown in Fig.6. The error components of $\lambda_{\text {iron }}$ are error of experimental data and of gap size between filler plate and iron shields. $\lambda_{\text {iron }}$ which are evaluated by average of $\lambda_{\text {iron }}$ for each reaction and considering above error components is $150 \pm 5 \mathrm{~g} / \mathrm{cm}^{2}$. This result is compared with previous studies ${ }^{14,15}$ for Moyer's formula, however the conditions of these studies are different as shown in Table 2. This value is in the medium of that of previous studies, $144 \mathrm{~g} / \mathrm{cm}^{2}$ by Stevenson et al. ${ }^{14)}$ and $188 \mathrm{~g} / \mathrm{cm}^{2}$ by Ban et al. ${ }^{15}$.

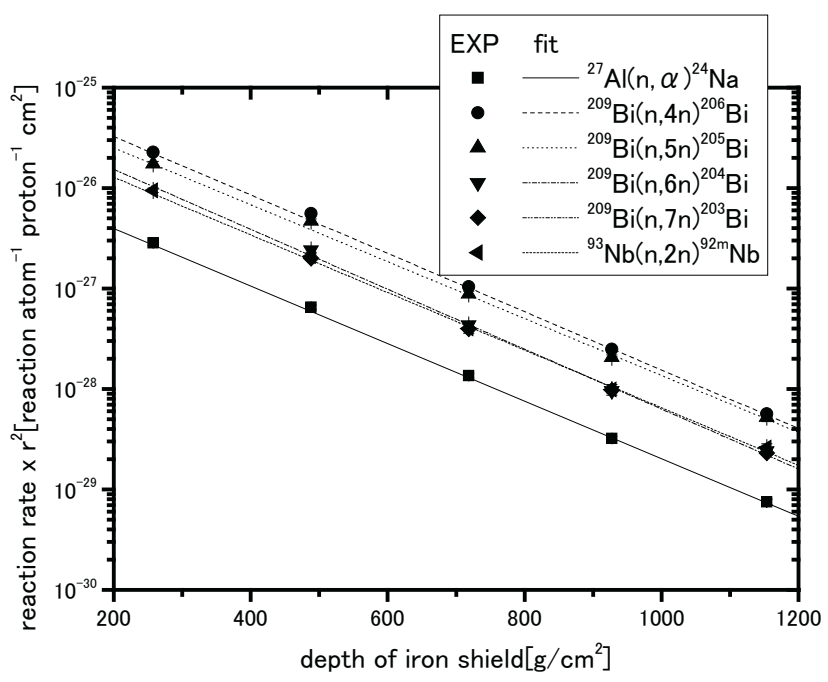

Fig. 6 The depth distribution of corrected reaction rate for 90 degree direction from beam axis.

Table. 2 The conditions of studies for Moyer model.

\begin{tabular}{ccc}
\hline & Projectile \\
Energy[GeV] & Target \\
\hline This work & 120 & inconel(Ni), Cu \\
Stevenson et al. $^{15)}$ & $5 \sim 30$ & $\mathrm{Al}, \mathrm{W}, \mathrm{Cu}$ \\
Ban et al. $^{16)}$ & 12 & $\mathrm{Pt}$ \\
\hline
\end{tabular}

\section{Application for the Moyer's formula}

The obtained reaction rates of ${ }^{27} \mathrm{Al}(\mathrm{n}, \alpha){ }^{24} \mathrm{Na}$ and

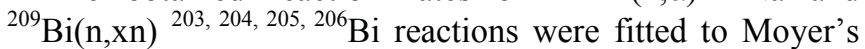
formula (following Eq.(1) for AG samples and Eq.(2) for CC samples, respectively).

$$
R=\frac{R_{0} e^{-b \vartheta} e^{\frac{d_{i r o n}}{\lambda_{i r o n} \sin \theta}}}{r^{2}}
$$

and

$$
R=\frac{R_{0} e^{-b \vartheta} e^{\frac{d_{i r o n}}{\lambda_{i r o n} \sin \theta}} e^{\frac{d_{c o n}}{\lambda_{c o n} \sin \theta}}}{r^{2}}
$$

Where $\mathrm{R}$ is reaction rate of sample, $\mathrm{R}_{0}$ is reaction rate at target position, $\mathrm{b}$ is parameter which indicates angular distribution, $\theta$ is angle from beam axis, $\mathrm{d}_{\text {iron }}$ is depth of iron shield, $d_{c o n}$ is depth of concrete shield, $\lambda_{\text {con }}$ is attenuation length for concrete shield. In this fitting, $\lambda_{\text {con }}$ is estimated as $114\left[\mathrm{~g} / \mathrm{cm}^{2}\right]$ from assumption that neutrons attenuate in proportion to geometrical cross section, $r$ is distance between target and samples.

Figs. 7 and 8 show comparison of measured reaction rate produced in $\mathrm{CC}$ and $\mathrm{AG}$ samples with reaction rates fitted by using Moyer's formula, respectively. 




Fig. 7 Comparison of measured reaction rate produced in CC samples with reaction rates fitted by using Moyer's formula.

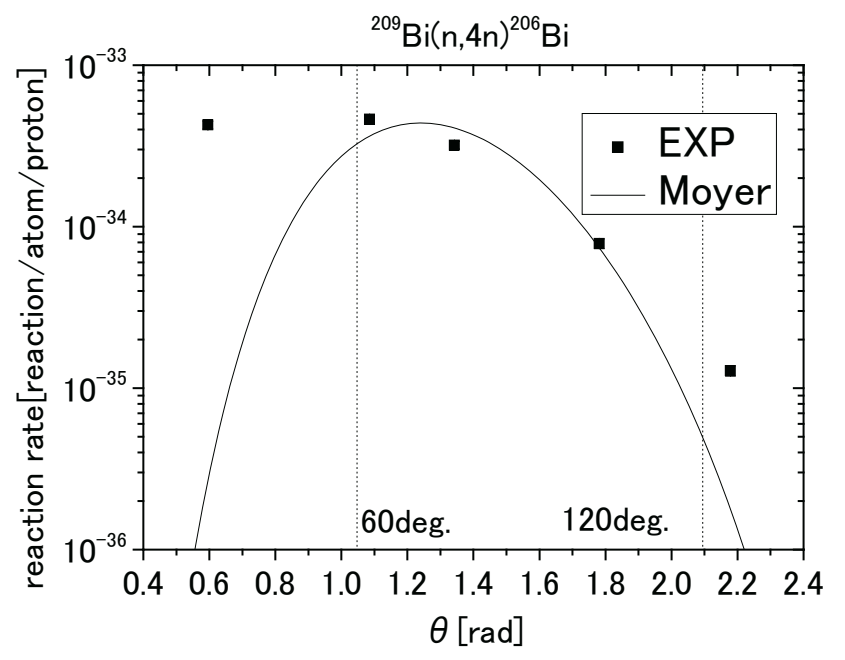

Fig. 8 comparison of measured reaction rate produced in AG samples with reaction rates fitted by using Moyer's formula.

In Figs. 7 and 8, shape of angular distribution of fitted reaction rates agree with that of measured reaction rates for $\theta$ $=60-120$ degrees. The angular distribution parameters, $\mathrm{b}$ which are obtained by fitting is 4.0 radian $^{-1}$. This value is larger than that of previous studies, 2.3 by Stevenson et al. ${ }^{14)}$ and 2.5 by Ban et al. ${ }^{15)}$.

In this ongoing study, absolute value of reaction rate could not be fitted by Moyer's formula, because the depth distribution of reaction rate in concrete shield have not be measured yet. The experiment for depth distribution measurement of reaction rate in concrete shield is planned.

\section{Conclusion}

The spatial distribution of reaction rate of samples which were placed on pbar anti proton target station were obtained. The attenuation length for iron was obtained from depth distribution of reaction rates in iron shield. The obtained reaction rates were also fitted to Moyer's formula. The shape of angular distribution of fitted reaction rates agree with that of measured reaction rates for $\theta=60$ - 120 degrees.

These experimental results will be useful as benchmark data to investigate the accuracy of various transport calculation codes.

\section{Acknowledgment}

This work is supported by grand-aid of ministry of education (KAKENHI 19360432) in Japan. Fermilab is a U.S. Department of Energy Laboratory operated under Contract DE-AC02-07CH11359 by the Fermi Research Alliance, LLC.

\section{References}

1) H. Nakashima, et.al, Nucl. Technol.168[2], 482 (2009).

2) N. Nakao, et al., Nucl.Sci.Eng. 124, 228 (1996).

3) H.Nakashima, et al., Nucl.Sci.Eng. 124, 243 (1996).

4) T.Nunomiya, et al., Nucl.Instrum.Meth. B179, 89 (2001).

5) N.Nakao, et al., Radiat.Prot.Dosim. 116, 553 (2005).

6) M.Awschalom, et al., Nucl.Instrum.Meth. 136, 521 (1976).

7) H.Nakashima, et al., Proc. of the Shielding Aspects on Accelerator, Target and Irradiation Facilities -SATIF6, SLAC, Apr. 10-12, 2002, p. 27 (2002).

8) N.Nakao, et al., Nucl.Instrum.Meth. A 562, 950 (2006).

9) N. V. Mokhov, , FERMILAB-FN-628 (1995), N.V. Mokhov, S.I. Striganov, Fermilab-Conf-07-008-AD (2007), Hadronic Shower Simulation Workshop AIP Proceedings 896.

10) B. J.Moyer et al., Proc. 1st Int. Conf. Shielding around High Energy Accelerators, Presses Universitaires de France, Paris (1962).

11) H. W. Patterson, R. H. Thomas, Accelerator Health Physics, Academic Press, New York (1973).

12) R. B. Firestone and V. S. Shirley,Eds. John Wiley and Sons,Inc,New york, Table of Isotopes, 8th edition (1996).

13) F. L. Bronson, J. Radioanal. Nucl. Chem., 255, 137 (2003).

14) G. R. Stevenson et al., Health Phys., 43, 13 (1982).

15) S. Ban et al., Nucl. Instum. Methods, 174, 271 (1980). 